\title{
BMJ Open Contributing factors that influence medication errors in the prehospital paramedic environment: a mixed- method systematic review protocol
}

\author{
Dennis Walker (D) , ${ }^{1}$ Clint Moloney, ${ }^{2}$ Brendan SueSee, ${ }^{3}$ Renee Sharples ${ }^{4}$
}

To cite: Walker D, Moloney $\mathrm{C}$, SueSee B, et al. Contributing factors that influence medication errors in the prehospital paramedic environment: a mixed-method systematic review protocol. BMJ Open 2019;9:e034094. doi:10.1136/ bmjopen-2019-034094

- Prepublication history and additional material for this paper are available online. To view these files, please visit the journal online (http://dx.doi. org/10.1136/bmjopen-2019034094).

Received 06 September 2019 Revised 23 November 2019 Accepted 27 November 2019

Check for updates

(C) Author(s) (or their employer(s)) 2019. Re-use permitted under CC BY-NC. No commercial re-use. See rights and permissions. Published by BMJ.

${ }^{1}$ School of Health and Wellbeing, University of Southern Queensland, Ipswich, Queensland, Australia

${ }^{2}$ School of Nursing and Midwifery, University of Southern Queensland, Toowoomba, Queensland, Australia

${ }^{3}$ School of Linguistics, Adult and Special Education, University of Southern QueenslandSpringfield Campus, Springfield, Queensland, Australia

${ }^{4}$ College of Health and Biomedicine, Victoria University, Melbourne, Victoria, Australia

Correspondence to

Mr Dennis Walker;

Dennis.Walker@usq.edu.au

\section{ABSTRACT}

Introduction There is limited reliable research available on medication errors in relation to paramedic practice, with most evidence-based medication safety guidelines based on research in nursing, operating theatre and pharmacy settings. While similarities exist, evidence suggests that the prehospital environment is distinctly different in many aspects. The prevention of errors requires attention to factors from the organisational and regulatory level down to specific tasks and patient characteristics. The evidence available suggests errors may occur in up to $12.76 \%$ of medication administrations in some prehospital settings. With multiple sources stating that the errors are under-reported, this represents significant potential for patient harm. This review will seek to identify the factors influencing the occurrence of medication errors by paramedics in the prehospital environment.

Methods and analysis The review will include qualitative and quantitative studies involving interventions or phenomena regarding medication errors or medication safety relating to paramedics (including emergency medical technicians and other prehospital care providers) within the prehospital environment. A search will be conducted using MEDLINE (Ovid), EBSCOhost Megafile Search, the International Committee of Medical Journal Editors trial registry, Google Scholar and the OpenGrey database to identify studies meeting this inclusion criteria, with initial searches commencing 30 September 2019. Studies selected will undergo assessment of methodological quality, with data to be extracted from all studies irrespective of quality. Each stage of study selection, appraisal and data extraction will be conducted by two reviewers, with a third reviewer deciding any unresolved conflicts. The review will follow a convergent integrated approach, conducting a single qualitative synthesis of qualitative and 'qualitised' quantitative data. Ethics and dissemination No ethical approval was required for this review. Findings from this systematic review will be disseminated via publications, reports and conference presentations.

\section{INTRODUCTION}

Paramedicine is an evolving profession, with paramedics now recognised as registered professionals within Australia. ${ }^{1}$ With expanding roles and increasing scopes of

\section{Strengths and limitations of this study}

- Given the limited range of evidence available on this topic, the mixed-method methodology allows a wider range of evidence to be included.

- This will be the first reliable systematic review focusing solely on medication error in the prehospital environment, and carried out by a multidisciplinary team inclusive of registered paramedics.

- Transformation of quantitative data to qualitised data makes the assessment of certainty using tools, such as GRADE (Grading of Recommendations, Assessment, Development and Evaluations), less reliable.

- Only studies published in English will be considered for the review.

practice among paramedics, the risk of medication errors occurring also increases. ${ }^{2}$

Medication error can be broadly defined according to the following criteria: administering the wrong medication; administering an incorrect dose of medication, both overdose or underdose; administration of medication at the wrong time (eg, repeating a dose too early or too late); administration of medication via the wrong route/method; administering medication that is inappropriate for the patient's condition due to the patient being allergic to the medication or there being a known or predictable clinical condition that will exacerbate side effects or cause an adverse reaction in combination with the medication. ${ }^{3-5}$

Factors that may contribute to error are broadly categorised into regulatory factors, organisational factors and factors associated with the work environment, interprofessional team, individual health care providers, specific tasks and the patient. ${ }^{67}$

Traditionally in medication safety research, the factors thought to be the cause of errors are violations of established procedures, 
indicative of carelessness, neglect, inattention or other reckless behaviour on the part of those personally involved. ${ }^{6}$ While these aspects of behaviour may occur in any workplace, errors still occur when procedures are carried out by competent and careful practitioners. Contemporary safety theory, much of which has originated from the airline industry, focuses on the effects of systems that may precipitate error or error producing conditions. ${ }^{67}$ The systems approach suggests that errors are a part of human nature and that safeguards should be put in place to prevent conditions that allow errors to occur or to potentially cause harm. ${ }^{6}$ These principles are evident in the many iterations of Reason's Swiss Cheese Model. ${ }^{6}$

Multiple evidence-based guidelines exist for the hospital, operating theatre and pharmacy environments, where the majority of existing research has been conducted. While these have been applied to prehospital practice, it must be recognised that the prehospital environment is distinctly different in many ways. Literature suggests that the main issues influencing paramedic medication errors are technical and environmental; citing excessive noise, distractions, poor lighting, unstable and sometimes dangerous environments and fatigue. ${ }^{8-11}$ These conditions result in increased physical and psychological pressures on paramedics when trying to make good judgements and follow safe medication administration practices. ${ }^{8-11}$ It is proposed that the number of medications administered to a single patient, along with the time spent treating the patient, as well as the relative complexity of the patient's condition also increases the likelihood of errors. ${ }^{2}$ Other sources cite medication storage, appearance and labelling as potential sources or error. ${ }^{8}$

Multiple sources in both the prehospital and hospital environments also discuss the under-reporting of medication errors specifically, or adverse events, of which medication errors are a subset. ${ }^{11-13}$ While the reporting of errors is a separate issue from the prevalence of the errors themselves, it does indirectly influence the occurrence of medication errors. The lack of root cause analysis of incidents and/or near misses leads to an inadequate understanding of and consequently the inability to address factors leading to those unreported errors. ${ }^{11}$

The limited evidence available estimates the rate of errors at as high as $12.76 \%$ in some prehospital environments. ${ }^{2}$ This figure represents medication errors in a cohort of critically unwell patients and cannot easily be applied to the workload statistics of Australian State Ambulance Services. In 2016-2017, Australian Ambulance Services responded to 1319552 emergency incidents (ie, responded to with lights and sirens activated). ${ }^{14}$ To demonstrate the potential enormity of this issue, however, if just $1 \%$ of these emergency incidents were considered to be similar to those in the study by Lifshitz et al. ${ }^{2}$ this could represent over 16800 medication errors per year. ${ }^{14}$ Such errors could include anything from significant errors (eg, wrong medication administered) to minor errors of timing (eg, a repeat dose not given at the appropriate time).$^{2-5}$ A clear picture of the factors influencing medication safety is required to guide further research into prevention strategies that could be applied to the prehospital environment.

\section{EXISTING SYSTEMATIC REVIEWS}

A search for systematic reviews, either in-progress or published, was conducted using PROSPERO, MEDLINE (PubMed), CINAHL, the Cochrane Database of Systematic Reviews, the Joanna Briggs Institute (JBI) Database of Systematic Reviews and Implementation Reports and the JBI Registered Systematic Reviews listings. The search found two existing systematic reviews relevant to this topic area:

In 2012, Bigham et al conducted a systematic review of the literature relating to patient safety, specific to the prehospital or emergency medical services (EMS) environment. This study extensively reviewed research reported in English, and published between 1999 and 2011. Of the 5959 articles identified, only 88 articles satisfied the inclusion criteria, of which 22 (25\% of included articles) were related to adverse events and medication errors. The majority of these articles focused on adverse events broadly rather than medication errors specifically.

There is a significant need to conduct a current review on medication errors, as the review by Bigham et al. ${ }^{8}$ is not specific to medication errors, and is now out of date. It is acknowledged that other areas of care addressed by Bigham et al. ${ }^{8}$ may be influenced by similar factors specific to the prehospital environment. Interventions, such as airway management, defibrillation and immobilisation, have their own specific risk profiles and bodies of evidence; it is the opinion of the authors that these areas of prehospital care deserve their own focused research.

The only other systematic review found was conducted by Hughes et al. ${ }^{15}$ This review, while more specific to medication errors and also more recent, fails to meet the methodological standards required by the Preferred Reporting Items for Systematic Reviews and Meta-analyses (PRISMA).${ }^{16}$ The outline of the selection process is lacking as per the PRISMA guidelines ${ }^{16}$ giving details of the inclusion and exclusion criteria, but non-specific details regarding the search strategy and actual numbers of articles included/excluded. There is no PRISMA flowchart outlining exactly how many articles were found. There is very limited evidence of appraisal of the articles and some lack applicability to the EMS environment generally (e.g., statistics from a paediatric medication error study ${ }^{17}$ are applied generally to all medication errors in EMS; a study regarding increased medication errors during transition from home to nursing home facilities has been applied to the EMS environment also). ${ }^{18}$

The focus of the review by Hughes $e t a l^{15}$ is also quite different from the proposed review. Hughes et $a l^{15}$ focused almost entirely on error, the taxonomy of EMS medication error and the application of the Human Factors Analysis and Classification System in this context. 
It is noted by Reason ${ }^{6}$ that the human behaviour may also be protective in a healthcare setting as opposed to the fallible weak link that facilitates error when system factors fail. Neither Bigham et $a l^{8}$ nor Hughes $e t a l^{15}$ have specifically mentioned protective factors within their reviews.

The objective of this review is to identify the factors influencing the occurrence of medication errors by paramedics in the prehospital environment.

\section{REVIEW QUESTIONS}

What contributory factors are known to influence medication errors in the prehospital paramedic environment?

This review seeks to identify the nature of known (actual or potential) or concealed medication errors in the prehospital paramedic environment. This review also seeks to define the exact context of medication errors within the prehospital environment, such that it could then be compared with other environments, such as hospitals, pharmacies and operating theatres in future research.

\section{INCLUSION CRITERIA \\ Participants}

Studies will be considered that include paramedics or prehospital health workers with a similar role description. This will include Emergency Medical Technicians and other descriptors of paramedics (eg, Advanced Care Paramedic, Flight Paramedic...) and studies addressing healthcare teams inclusive of paramedics (eg, retrieval teams).

\section{Phenomena of interest}

This review will consider studies that explore medication safety or medication errors.

\section{Context}

Any studies that address medication errors or medication safety concepts within the prehospital environment will be considered for inclusion.

\section{Types of studies}

Both quantitative and qualitative studies will be considered for inclusion. This will include experimental and quasi-experimental study designs (randomised controlled trials, non-randomised controlled trials, before-and-after studies and interrupted time-series studies), analytical observational studies (prospective and retrospective cohort studies, case-control studies and analytical cross-sectional studies), descriptive observational study designs (case series, individual case reports and descriptive cross-sectional studies) and studies that focus on qualitative data (including, but not limited to, designs such as phenomenology, grounded theory, ethnography, action research and feminist research). Only studies published in English will be considered for inclusion. Studies published from database inception to the present will be included.

\section{METHODS}

The proposed systematic review will be conducted in accordance with the Joanna Briggs Institute Methodology for Mixed-Methods Systematic Reviews and the PRISMA reporting guidelines. ${ }^{16} 19$

\section{Search strategy}

The search strategy will aim to locate both published and unpublished studies with no date limits (ie, from database inception to the present). An initial limited search of MEDLINE was undertaken to identify articles on the topic. The text words contained in the titles and abstracts of relevant articles, and the index terms used to describe the articles were used to develop a full search strategy for MEDLINE (see online supplementary appendix I). The search strategy, including all identified keywords and index terms, will be adapted for each included information source. The reference lists of all studies selected for critical appraisal will be screened for additional studies. Initial searches will commence on 30 September 2019.

\section{Information sources}

MEDLINE (Ovid), EBSCOhost Megafile Search, the International Committee of Medical Journal Editors trial registry, Google Scholar and the OpenGrey database.

\section{Study selection}

Following the search, all identified citations will be collated and uploaded into ENDNOTE V.X9.2 and duplicates removed. Titles and abstracts will then be screened by two independent reviewers for assessment against the inclusion criteria for the review. Potentially relevant studies will be retrieved in full and their citation details imported into Covidence (or comparable systematic review management software) for further management of the data. Training will be provided on the use of the review management software to all reviewers. Study selection and data extraction processes will be piloted and tested for accuracy prior to these processes being conducted.

The full text of selected citations will be assessed in detail against the inclusion criteria by the two independent reviewers. Reasons for exclusion of full-text studies that do not meet the inclusion criteria will be recorded and reported in the systematic review. Any disagreements that arise between the reviewers at each stage of the study selection process will be resolved through discussion or with a third reviewer. The results of the search will be reported in full in the final systematic review and presented in a PRISMA flow diagram (see figure 1). ${ }^{16}$

\section{Assessment of methodological quality: quantitative}

Eligible studies will be critically appraised by the two independent reviewers at the study level for methodological quality using the applicable standardised critical appraisal instruments from the Joanna Briggs Institute for experimental, quasi-experimental and observational studies. ${ }^{19}$ The authors of papers will be contacted to request missing or additional data for clarification, where required. Any disagreements that arise will be resolved 


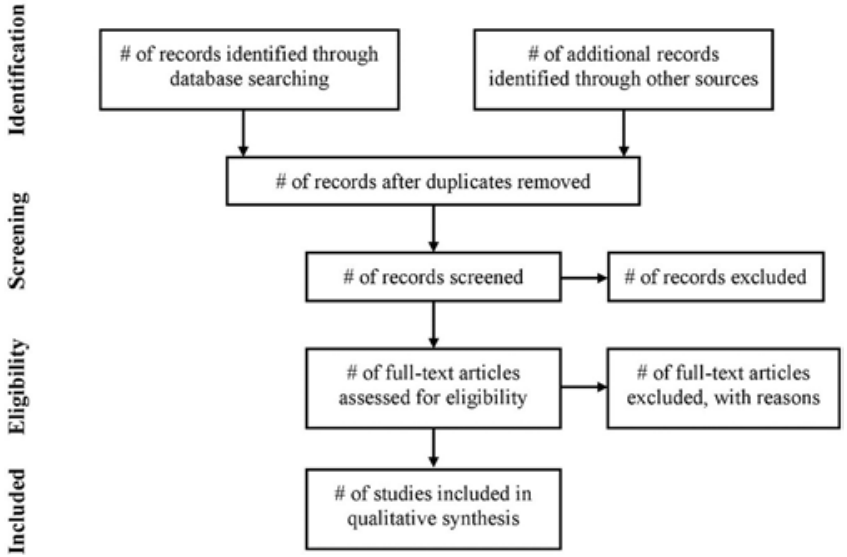

Figure 1 Preferred Reporting Items for Systematic Reviews and Meta-analyses (PRISMA) flow diagram in figure 1 outlines the process of article selection as per the PRISMA guidelines. ${ }^{16}$ This starts with the results of the initial database searches, and then the total number of articles after removal of duplicates. It further details the number of articles excluded during screening of titles and abstracts, and then again on assessment of the full-text articles.

through discussion, or with a third reviewer if a consensus cannot be reached. The results of critical appraisal will be reported in narrative form and in a table.

All studies, regardless of the results of their methodological quality, will undergo data extraction and synthesis (where possible). The results of the critical appraisal will be appended to the review.

\section{Assessment of methodological quality: qualitative}

Eligible studies will be critically appraised by the two independent reviewers for methodological quality using the standard Joanna Briggs Institute Critical Appraisal Checklist for Qualitative Research. ${ }^{19}$ Any disagreements that arise between the reviewers will be resolved through discussion, or with a third reviewer if a consensus cannot be reached. The results of critical appraisal will be reported in narrative form and in a table.

All studies, regardless of the results of their methodological quality, will undergo data extraction and synthesis (where possible).

\section{Data extraction}

Data will be extracted from studies included in the review by two independent reviewers using a modification of the Joanna Briggs Institute data extraction tool (see online supplementary appendix II) and following a convergent integrated approach. ${ }^{19}$ The data extracted will include specific details about the populations, context, culture, geographical location, study methods and the phenomena of interest relevant to the review objectives. Quantitative outcome data will be extracted composed of the numerical, data-based results of descriptive and/or inferential statistical tests. The findings of qualitative studies, and their illustrations, will be extracted and assigned a level of credibility. Any disagreements that arise between the reviewers will be resolved through discussion or with a

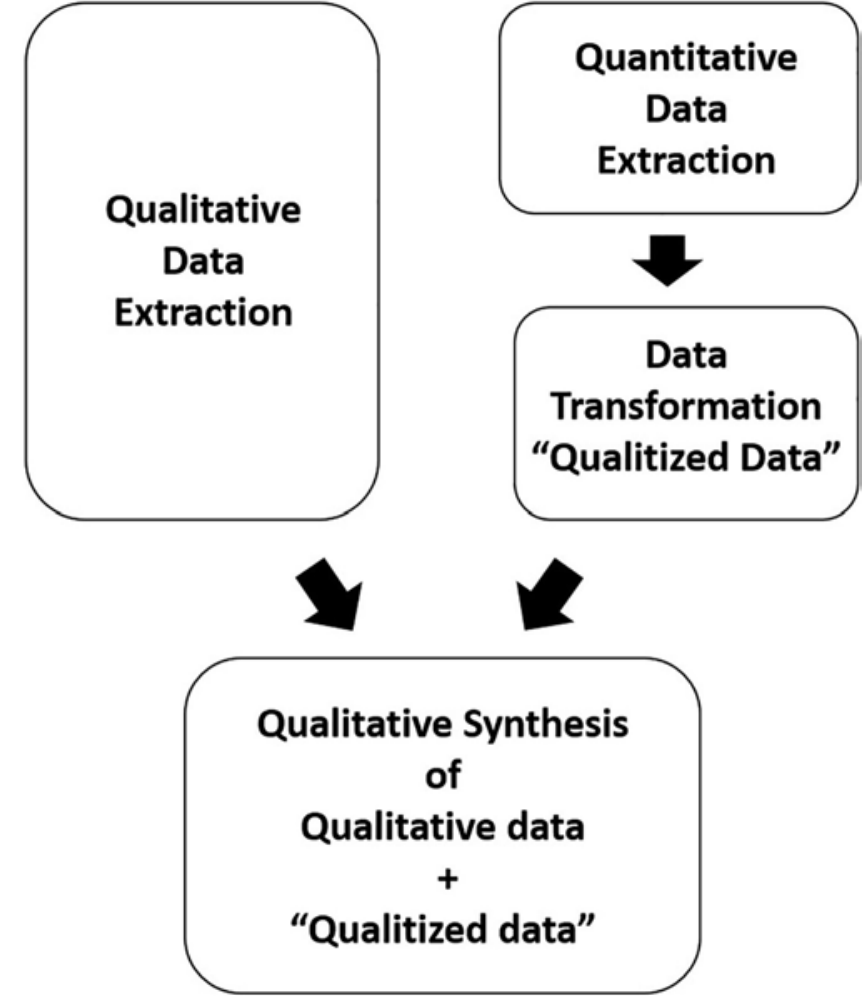

Figure 2 Data transformation in figure 2 graphically outlines the process of data transformation as per the convergent integrated methodology described by Lizarondo et al. ${ }^{19}$ This process involves the conversion of numerical quantitative data into text-based 'qualitised' data, which can then be combined with other qualitative data as part of an overall qualitative synthesis.

third reviewer. The authors of papers will be contacted to request missing or additional data, where required.

\section{Data transformation}

Quantitative data will be converted into 'qualitised data' according to the JBI Convergent Integrated Approach (see figure 2). ${ }^{19}$ The numerical data will be transformed into textual descriptions or narrative interpretations of the quantitative results so as to respond directly to the review question.

\section{Data synthesis}

The review will use a convergent integrated approach, as per the JBI methodology for mixed-methods systematic reviews. ${ }^{19}$ The qualitised data and qualitative data will be categorised and aggregated based on similarity of themes to produce a set of integrated findings. These will be presented in the form of line of action statements.

\section{PATIENT AND PUBLIC INVOLVEMENT}

There are no plans for patient or public involvement with the design, conduct, reporting or dissemination this systematic review. There are no patients involved in the proposed study. 


\section{ETHICS AND DISSEMINATION}

Findings from this systematic review will be disseminated via publications, reports and conference presentations. As the findings of this review will establish the key factors influencing medication error in the prehospital environment, this will form the basis for future guideline development and research into the prevention of medication error within the prehospital environment.

Contributors DW was responsible for the design of the review and protocol, supported by CM and BS as research supervisors. All authors (DW, CM, BS and RS) have contributed to the development and editing of the protocol.

Funding The corresponding author has received support from the University of Southern Queensland as a PhD student.

Competing interests None declared.

Patient consent for publication Not required.

Ethics approval There is no ethical approval required for this review.

Provenance and peer review Not commissioned; externally peer reviewed.

Open access This is an open access article distributed in accordance with the Creative Commons Attribution Non Commercial (CC BY-NC 4.0) license, which permits others to distribute, remix, adapt, build upon this work non-commercially, and license their derivative works on different terms, provided the original work is properly cited, appropriate credit is given, any changes made indicated, and the use is non-commercial. See: http://creativecommons.org/licenses/by-nc/4.0/.

ORCID iD

Dennis Walker http://orcid.org/0000-0003-0322-845X

\section{REFERENCES}

1 Paramedicine Board of Australia. Registration [Internet]. Australian Health Practitioner Registration Agency, 2019. Available: https:// www.paramedicineboard.gov.au/registration.aspx [Accessed 28 Aug 2019].

2 Lifshitz AE, Goldstein LH, Sharist M, et al. Medication prescribing errors in the prehospital setting and in the ED. Am J Emerg Med 2012;30:726-31.

3 Crossman M. Technical and environmental impact on medication error in Paramedic practice: a review of causes, consequences and strategies for prevention. Australasian Journal of Paramedicine $2009 ; 7$.
4 Leufer T, Cleary-Holdforth J. Let's do no harm: medication errors in nursing: Part 1. Nurse Educ Pract 2013;13:213-6.

5 Roughhead L, Semple S, Rosenfeld E. Literature review medication safety in Australia 2013. Sydney: Australian Commission on Safety and Quality in Health Care, 2013.

6 Reason J. Human error: models and management. BMJ 2000;320:768-70.

7 Vincent C, Taylor-Adams S, Chapman EJ, et al. How to investigate and analyse clinical incidents: clinical risk unit and association of litigation and risk management protocol. BMJ 2000;320:777-81.

8 Bigham BL, Buick JE, Brooks SC, et al. Patient safety in emergency medical services: a systematic review of the literature. Prehosp Emerg Care 2012;16:20-35.

9 Atack L, Maher JEmergency medical and health providers' perceptions of key issues in prehospital patient safety. Prehosp Emerg Care 2010;14:95-102.

10 Fairbanks RJ, Crittenden CN, O'Gara KG, et al. Emergency medical services provider perceptions of the nature of adverse events and near-misses in out-of-hospital care: an ethnographic view. Acad Emerg Med 2008;15:633-40.

11 Wolf ZR, Hughes RG. Error Reporting and Disclosure. In: Hughes $\mathrm{R}$, ed. Patient safety and quality: an evidence-based Handbook for nurses. Rockville (MD): Agency for Healthcare Research and Quality (US), 2008.

12 Hobgood C, Weiner B, Tamayo-Sarver JH, et al. Medical error identification, disclosure, and reporting: do emergency medicine provider groups differ? Acad Emerg Med 2006;13:443-51.

13 Vilke GM, Tornabene SV, Stepanski B, et al. Paramedic self-reported medication errors. Prehosp Emerg Care 2007;11:80-4.

14 Australian Government Productivity Commission. Report on government services 2018, 2018. Available: https://www.pc.gov. $\mathrm{au} /$ research/ongoing/report-on-government-services/2018/health/ ambulance-services [Accessed 28 Aug 2019].

15 Hughes AM, Sonesh S, Zajac S, et al. Leveraging HFACS to understand medication error in emergency medical services (EMS). Proc Hum Factors Ergon Soc Annu Meet 2013;57:1688-92.

16 Moher D, Liberati A, Tetzlaff J, et al. Preferred reporting items for systematic reviews and meta-analyses: the PRISMA statement. PLoS Med 2009;6:e1000097.

17 Hoyle JD, Davis AT, Putman KK, et al. Medication dosing errors in pediatric patients treated by emergency medical services. Prehosp Emerg Care 2012;16:59-66.

18 Desai R, Williams CE, Greene SB, et al. Medication errors during patient transitions into nursing homes: characteristics and association with patient harm. Am J Geriatr Pharmacother 2011;9:413-22.

19 Lizarondo L, Stern C, Carrier J. Chapter 8: Mixed methods systematic reviews. In: Aromataris E, Munn Z, eds. Joanna Briggs Institute Reviewer's Manual. The Joanna Briggs Institute, 2017. https://reviewersmanual.joannabriggs.org/ 